Historic, Archive Document

Do not assume content reflects current scientific knowledge, policies, or practices. 


\section{Supplement to my "Master" Catalog}

Please insert this supplement in the catalog and keep with it as it contains changes in prices, new varieties, etc.

12 bulbs at price of 10, postage paid. My bulbs this yearare the finest crop I ever harvested.

77 South Ave.

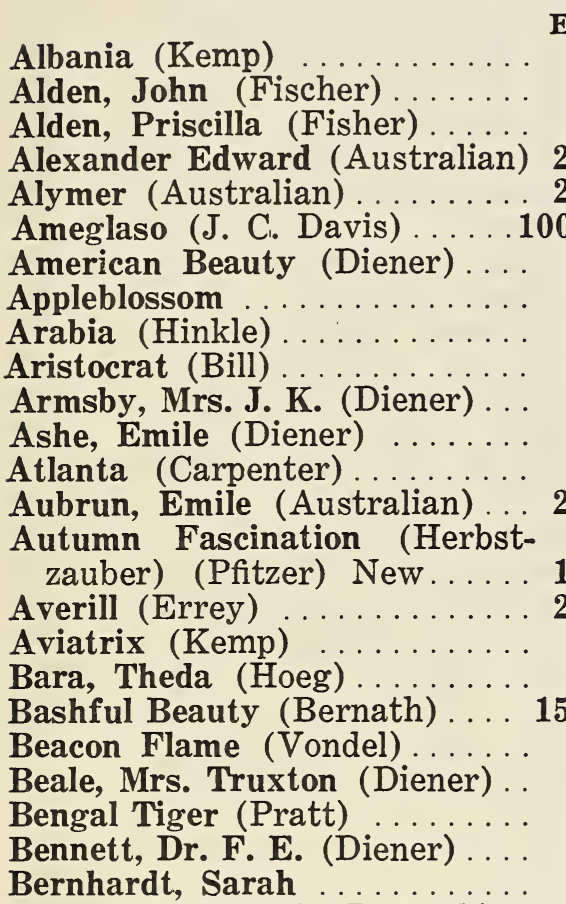

Bernath, Elizabeth (Bernath) . 10.00

Black Joe (Prestgard)

Black Pansy (Kunderd)

Bolt, Ben (Prestgard) ....... . .20

Bothin, Genevieve (Diener) . . . . .35

Bothin, Mrs. H. E. (Diener) . . . . .25

Break 0'Day (Bill)

Buffalo Bill (Dr. Hoeg)

Bunce, Allen V. (Bill)

Burke, Mrs. Mary Stearns

(Diener)

California Canary (Salbach) . 1.00

California Giant (Diener) ..... .50

Cameo (Bill)

Captain Boynton (Boynton) ... .20

Capitaine Ferber (Lemoine) . . . .25

Carbone, J. A. (Diener) . . . . 1.00

Caretta (John C. Davis) ...... 1.00

Carmen Sylva (Prestgard) ..... . .10

Caruso (New Holland) cerovarvid

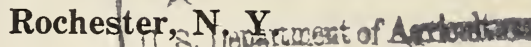

Casa Blanca (Lemoine) . . . . . $\begin{aligned} & \text { Each } \\ & \mathbf{1 . 0 0}\end{aligned}$

Catherina (Velthuys)

.25

Cerinthe (Australian)

2.50

Challenger (Kunderd)

.10

Chancellor (Errey)

2.00

Charlemagne (Lemoine)

.10

Chateau Thierry (Vos) ....... .10

Chris (Gruellmans) .......... .10

Claremont (Salbach)

.30

Clark, Mrs. Wm. E. (Fischer) .. .15

Coleman, Catherine (Coleman) . $\quad .40$

Coleman, Joe (Coleman) ...... .10

Concolor (Lemoine) . . . . . . . . .25

Constance (Errey) . . . . . . . . . 2.00

Coral Star (Bernath) . . . . . . . . 10.00

Corunna (Kunderd) .......... .15

Costa Rica (Diener) . . . . . . . . . .25

Coulter, John Merle (Diener) . . .25

Crimson Glow (Betscher) ...... .10

Crystal (John C. Davis) ....... $\quad .50$

Cynthia (Dr. Hoeg) . . . . . . . . 50

Dainty Lady .............. 1.00

Dawn, Hazel (Dr. Hoeg) ...... $\quad .30$

Day, Ella (Carpenter 1926) . . . 10.00

Day, Lynda (Carpenter) . . . . . . .15

Defiance (Kunderd) .......... .10

De Luxembourg, Adelaide (Holland) $\mathrm{New}$

De Poy, Ada (Salbach)

Dickens, Charles (New Holland) 1.50

Diener's American Beauty (Diener)

Diener, Richard (Diener)

Dorothy Ann (J. K. R.)

Douglas, Mrs. Leon (Diener) . . . $\quad .50$

Duches of York ............ . .35

DuTailles, Jean (Lemoine) ..... . .25

Early Snowflake (Kemp) . . . . . . . 10

Early Sunrise (Velthuys) . . . . . . .25

Eberius, Anna (Diener) ...... . .10

Edison, Thomas ........... . .40

Elaine (Bill) . . . . . . . . . . . . .30

Eldora (Kemp) ........... . 80

Elf (Diener) .............. .25

1.00 Elora (Burbank) ........... 10 
Ensign (Errey)

2.00

2.00

.10

.35

.15

.15

Ewbank, Rev.

Farmer's Wife (Rugowski)

Fastidious (Bill)

Firy Knight (Hopman)

Fischer, Sophie (Fischer)

Flaming Sword

Flossie (Carpenter)

Foch, Marshal (Kunderd)

Ford, Henry (Diener)

Franklin, Miss Helen (Kunderd)

Friend of Mine (Carpenter 1926)

Fritzjof (Krelage)

General Langlois (Lemoine).

Genesee (J. C. Davis)

Gettysburg (Dr. Hoeg)

Giant Nymph (Coleman)

Gladdie Boy (Bill)

Glendale (W. B. Davis)

Gloriosa (J. C. Davis)

Glory of the Night (Cushman)

Gluck, Alma

Goehl, Henry C. (Fischer)

Gold (Hoeg)

Gold Eagle (Austin)

Gold Elsie (Fischer)

Golden Emblem (J. C. Davis) .

Golden Measure (Kelway)

Golden Swallow (Austin)

Golden Vliess (Pfitzer)

Graetz, Lena (Graetz)

Grace (Frazier) 1925

Greig, Edouard (Lemoine)

Grotz, Trudel

Hanks, Nancy (Salbach).

Harbinger (Sanford)

Harlem Valley (Bernath)

Hauff, Mrs. Dr.

Heart of Juanita (Diener)

Heavenly Blue (New Holland)

Herada (Austin)

Herbstzauber (Autumn Fascination) (Pfitzer)

Herodion (Errey)

Hinkle, Mrs. C. R. (J. C. Davis)

Hobbs, Col. (new)

Hulot, Baron J. (Lemoine)

Imperialis (Mrs. Hoeg)

Idamae (Salbach)

Illuminator (Hinkle)

Imperator (Heemskerk)

3.00

.20

.10

.35

.15

3.00

.35

2.00

.10

.15

.75

.10

.50

.50

1.00

.50

2.50

1.00

20.00

.10

.40
Iremel (Errey) . . . . . . . . . 2.00

Isis . . . . . . . . . . . . . . . . .25

Jackson, Dr. R. T. (Fischer) . . . $\quad .10$

Jap Lady (Prestgard) . . . . . . . . .75

Jewell (Prim.) (Zee) ........ . .15

Joerg's White (Joerg) . . . . . . . 1.25

Jordan, David Starr (Diener) . . $\quad .50$

.30 June Delight (Kunderd) . . . . . . .15

.10 Juniata (Kemp) . . . . . . . . 5.00

.75 Kent, Mrs. William (Diener) .. .25

.40 Kent, Thos. T. (Diener) ...... .25

1.00 King, Mrs. Francis (Coblentz) . 05

$.25 \quad$ King Pearl (Errey) . . . . . . . . 2.00

.50 King Tut (J. C. Davis) . . . . . . 100.00

.05 Kirtland, Evelyn (Austin) ..... .10

.50 La Beaute . . . . . . . . . . . . . . .50

.25 Lacinatus (Kunderd) . . . . . . . 1.00

.25 La Comete (Errey) . . . . . . . . 2.00

.15 La Couronne (Lemoine) . . . . . . .25

.50 Lady Byng (Gilchrist) . . . . . . . .50

.15 La Gaiete (Holland, new) . . . . 2.00

1.00 LaLuna (Groff) . . . . . . . . . . 50

.10 Lanphiere, Fay-Miss America (E. L. Williams)

La Serene (Wing)

Lavender Bride (Bride)

.20

Lavender Brine (Bride)

Lavender Princess (Kingsley) . . . .15

Laverne (Houdyshel)

Lavinia (Errey)

.50

Le Cid (Lemoine) ......... . .25

Lefebre, Eugene (Lemoine) . . . . .25

Lemoine, Henri (Lemoine) . . . . .25

Leota (Coleman) ........... $\quad .20$

Lilac Wonder (Gruellmans) . . . . $\quad .75$

Lilywhite ................ 10

L'Immaculee ............. .10

Lind, Jenny (Ruffled) (Hoeg) . . .20

Lindenow (Errey) ......... 2.00

Lois Mary (Carpenter 1926) ... .25

Lockwood, Arthur (Salbach) . . . .25

Lohrman, Mrs. Richard (Diener) 1.00

London, Jack (Diener) ....... . .25

Longfellow (Prestgard) ...... . .50

Loretta (Breeswine) . . . . . . . 1.50

Lorice (Kemp) . . . . . . . . . 1.50

Los Angeles (Houdyshel) . . . . . 20

1.50 Lost Purple (Bernhardt) ..... . .25

2.00 Lottie Belle (Carpenter) . . . . . .15

2.50 Louise (Wright) . . . . . . . . .15

.50 Loveliness ................ . .10

.10 Lucette (Bill) . . . . . . . . . . . . .10

.50 Lucie (Hopman) . . . . . . . . . . .15

.25 Lucius (Fischer) . . . . . . . . .50

.20 Madison, Miss (Boynton) ..... .20

Magnolia 
Maiden's Blush (Prim.) (Gruell mans)

Maine (Vos)

10.00

1.00

.15

1.00

.50

.75

5.00

2.00

.15

.15

.10

.40

.25

.05

.50

.75

.10

2.00

.10

1.00

.15

.25

.20

.05

.25

.50

.10

.10

2.00

.20

1.00

.05

.10

.25

.50

1.00

3.00

Peace (Groff)

Pearl of California (Kingsley) . $\mathbf{5 . 0 0}$ Medium-3.50 Small-2.50

Pendleton, Mrs.Frank (Kunderd)

Persia

Peters, Mrs. F. C. (Fischer)

Pfitzner, Dr. H. (Pfitzer) New $\mathbf{1 . 0 0}$

Pfitzer, Paul

1.50

Pfitzer's Triumph

Pfitzer, Wilhelm, Memory of (Pfitzer)
Phipps, W. H. (Diener)

Pickford, Mary (Kunderd)

Pinkie (J. C. D.)

Pink Perfection (Hopman)

Pink Wonder (Kemp)

Pippin (Bill)

Plummer, Sidney (Salbach)

Pollyanna (Prestgard)

Prestgard, Mrs. Kristian

$$
\text { (Prestgard) }
$$

Pride of Lancaster (Kunderd)

Pride of Rochester (J. C. Davis)

Primadonna (Fischer)

Primunella (Ruffied) (Kunderd)

Prince of Wales (Van Zanten)

Priority (Bill)

Purest of All

Purple Glory (Kunderd)

Purple Perfection

Pythia (Kunderd)

Quinton (Kemp)

Radium (Vos)

Rags (New-Just Out)

Rattray, Mrs. T. (Errey)

Reah (Salbach)

Red Emperor (Groff)

Red Giant (Biggard)

(No. 2 Bulbs)

Red Star

Remembrance (Kunderd)

Renown (Errey)

Richardson, Mrs. Willard (Diener)

Romance (Kunderd)

Roodkapje

Rose Ash (Diener)

Rosemary (Bales)

Rose, Miss T. (Fischer)

Rose Mist (Fischer)

Rose, Precose

Rouget De Lisle

Rubini (Heemskerk)

Ruffolace (Kemp)

Rugowski, Miss Cornelia (J.K.R.) 1.00

Sacajawea (Speelmon) ....... 3.50

Salmon Beauty (Kunderd) . . . . . $\quad .10$

San Anselmo (Diener) . . . . . . . .10

Saraband (Salbach) . . . . . . . .50. Sausalito (Diener) ......... .25

Scarlet Princeps (Kunderd) . . . $\quad .15$

Scarlet Wonder (Cowee) ..... . .25

Scarsdale (Cowee) .......... .25

Schneider, Camillo (Primulinus
.10

.15

.10

10

.15

.50

.10

.10

.10

.30

.15

1.00

.50

.10

.50

.35

.15

.15

.12

.15

3.00

.25

.25

1.00

.10

.25

1.00

.20

3.00

.15

5.00 10 50 
Each

Seedling $67 \mathrm{G} 42$ (W. F . Walsh) 2.00

Sentinel (Errev)

Shaylor, E. J. (Kunderd)

Shiela (Coleman)

Sibyl (Bill)

Sirene (Carpenter 1926)

Sisson, Mrs. P. W. (Coleman) . 20.00

Smiling Morn (Bernhardt) .... 1.00

Smith, Byron L. (Kunderd) . . . . $\quad .15$

Souvenir (Gravereau) . . . . . . . .15

Starbright (Bales) .......... 1.00

Sunburst (J. C. Davis) . . . . . . 2.00

Sunnymede (Fischer) ....... $\quad .40$

Sunset (Bernath) ......... 1.00

Sunset Glow (Gibbs) ........ . .15

Surprise (J. C. Davis) 1925 ... $\quad .50$

Sweetheart (Kemp) ........ . .25

Sweet Lavender (Coleman) . . . . $\quad .10$

Symmes, Frank J. (Diener) . . . . $\quad .10$

Tabor, Elizabeth (Hinkle) . . . . . .15

Tacoma (Martin) ........... 1.00

Talmadge, Norma (Dr. Hoeg) . 1.00

Tenny, Jean (Errey) . . . . . ... 2.50

Theda (Bill) ...............15

The Orchid (Sprague) ....... 2.50

Tiplady, Alice (Prim.) (Kunderd)

Titan (Errey)

Titanic (Hoeg)

.10

2.00

Triomphe de Caen (Vilmorin)

Triumphator (Krelage)

Trojana (Bill)

Twilight (Kunderd)

Twin Fires (Fischer)

Tyrian Beauty (Kunderd)

Van Beyren, Jacoba

(Heemskerk)

Van Konynenburg, Mrs.

Vanity (Bill)

Van Rappard, M.

Vaughan's White (Vaughan)

Veilchenblau (Pfitzer)

Venus (Bill)

Vesta Tilly (Krelage)

Victor (Errey)

Viola (Holland)

.15

1.00

1.00

.15

.20

.50

.15

Volkert, Karl

Walsh, Mrs. John R. (Diener) .

Wanita (Martin)

10.00

.15

.50

.50

1.50

.25

.10

2.00

1.00

1.00

.50

2.50

West, Sylvia (J. K. R.) 1927.500 .00

White City (Jonkheer)
White Giant (Van Meerbeck)

Each

.10

White Wonder (Kemp) . . . . . . . . 10

Wilbrink (Hopman) ........ $\mathbf{. 0 5}$

Winona (J. C. Davis) . . . . . . 1.00

Wisconsin (Vos) .......... $\quad .10$

Wolverine (Vos) . ............ $\quad .10$

Yellow Gem (Kunderd) . . . . . . $\quad .10$

Yellow Taxie (J. K. R.) . . . . . . $\quad \mathbf{. 5 0}$

Yellow Treasure (Austin) ..... $\quad .15$

Yosemite (Diener) . . . . . . . . . .50

Yvonne ................. $\quad .25$

Zang, Gretchen (Austin) . . . . . .05

Zang, Tycko (Austin) ....... .50

Zebra (Bernath) ......... 1.00

\section{NEW VARIETIES}

Amberosa

A new 1928 introduction of marvelous growth and beauty and is a fit variety to be grown in any garden. Outside of petals rose doree, amber yellow centre suffused with rose doree, no throat markings, has five to six fully open flowers each $41 / 2$ to $5 "$ across, correct placement and a strong tall spike. Substance is good and makes a grand exhibition or commercial flower, midseason. John says this is a good one.

Bobby Douglas (Rademacher) $\$ 2.50$ Exhibited at the Hartford Show under seedling No. 336 and attracted more attention than any other variety on account of its beautiful formed, extra large pure white flowers. It rates 96 in the Exhibition Gardens. Flowers are well placed on a long, straight spike and is as near perfection as any good pure white flower has ever come. You will be proud to grow this one. Bulbs $1 \frac{1}{4}$ or larger.

Peacock Blue (Reiman) .........10 A new color in gladiolus and is very attractive in the garden.

\section{SELECTED MIXED BULBS}

$\begin{array}{lllll}.10 & \text { Per } 100 & \$ 2.50 & \text { Per } 1 \text { doz. } 50 \mathrm{c}\end{array}$

Dahlias

Choice Dahlia tubers, all new, high priced varieties. Very large flowers, sure to grow, 25c each. Many worth $\$ 2.00$. You will be satisfied. 